\title{
Physical fatigue prediction based on heart rate variability (HRV) features in time and frequency domains using artificial neural networks model during exercise
}

\author{
Zulkifli Ahmad 1 2, Mohd Najeb Jamaludin 2, Ummu Kulthum Jamaludin 1 \\ 1 Human Engineering Group, Faculty of Mechanical and Manufacturing Engineering, \\ Universiti Malaysia Pahang, 26600, Pekan, Pahang, Malaysia. \\ 2 Sports Innovation and Technology Centre, Institute of Human Centered Engineering, \\ School of Biomedical Engineering and Health Sciences, Faculty of Engineering, \\ Universiti Teknologi Malaysia, 81310, Skudai, Johor, Malaysia. \\ zulkola84@gmail.com / kifli@ump.edu.my
}

\begin{abstract}
:
Awareness on fatigue level is important for people in order to understand their physiology in daily activities. This situation become more critical when involving physical exercise and reach the maximum threshold fatigue which can lead to injury. Additionally, sedentary people become the most group who is difficult to understand and know their fatigue condition based on feeling compared to the recreational exercise people and sports athlete. Therefore, this study is aims to help sedentary to predict the level of fatigue based on HRV features using artificial neural network (ANN). Eighteen sedentary peoples who are volunteer to participated in this study required to perform fatigue-induced protocol to achieve the heart rate maximum (HRmax). Those participants were run on the treadmill with speed intensities from $4 \mathrm{~km} / \mathrm{h}$ to $12 \mathrm{~km} / \mathrm{h}$ depends on their ability. During running, single-lead ECG was attached on the chest by using $\mathrm{Ag} / \mathrm{AgCl}$ wet electrodes. The raw signals which accumulate together with noise and motion artefacts were then filtered in 4th order Butterworth filter. A new signal of HRV was used to analyze by extracting the features in each level of fatigue based on Edward's Method zones. Eight features of time and frequency domains were selected in the neural network as input and predicts the fatigue zones as an output. HRV and HRmax were found as significant parameters to detect fatigue by differentiate its pattern in pre and post exercise. The results reveal that the prediction model with accuracy as high as $80.6 \%$ in the output of five fatigue classes. The results presented here may facilitate improvements in identifying the level of fatigue based on prediction algorithm compared to the RPE method during physical exercise.
\end{abstract}

Keywords: Prediction; Fatigue; HRV; ECG; ANN 


\section{Acknowledgement}

This work was supported by the Universiti Malaysia Pahang under the Research University Grant, RDU190322. Also, Universiti Teknologi Malaysia which providing facilities and equipment for experimental setup. 